\title{
3D multi-directional sensor with pyramid mirror and structured light
}

\author{
Jing Xu ${ }^{\text {a,b,* }}$ Peng Wang a Youyou Yao ${ }^{\text {a }}$ Shuntao Liu ${ }^{\mathrm{c}}$ Guanglie Zhang ${ }^{\mathrm{d}}$ \\ ${ }^{a}$ State Key Laboratory of Tribology, and Department of Mechanical Engineering, Tsinghua University, Beijing, 100084, China \\ ${ }^{\mathrm{b}}$ Beijing Key Lab of Precision/Ultra-precision Manufacturing Equipment and Control, Beijing 100084, China \\ ${ }^{\mathrm{c}}$ AVIC Chengdu Aircraft Industrial (Group) Co., Ltd, Chengdu, Sichuan, 610092, China. \\ ${ }^{\mathrm{d}}$ Shenzhen Academy of Robotics, Shenzhen, Guangdong, 518057, China.
}

\begin{abstract}
Structured-light-based 3D multi-directional sensors are widely used due to their flexible field of view (FOV), which is a significant benefit. Nevertheless, existing 3D multi-directional sensors based on linear lasers are only capable of measuring a slice of a 3D scene at low resolution. In addition, other 3D multi-directional sensors are capable of measuring 3D scenes by shooting 2D structured light patterns in different directions through curved mirrors. However, the projected and captured structured light patterns are blurred due to the optical aberration property of curved mirrors, resulting in inaccurate 3D reconstruction. This paper proposes a 3D, high-resolution, high-accuracy, real-time multi-directional sensor that utilizes pyramid mirrors to decompose the FOVs into different directions and reduce the optical aberration phenomenon. The mathematical model of the 3D sensor is presented, and the FOV is analyzed. A dual-frequency phase-shifting fringe pattern is used for 3D reconstruction in real time. Moreover, the sensor is calibrated using a planar circle calibration gauge and the Householder reflection constraint. Finally, the experimental results demonstrate that the measurement error of the 3D multi-directional sensor is less than $1 \mathrm{~mm}$ and $0.03 \mathrm{rad}$, thus verifying the method's feasibility.
\end{abstract}

Key words: multi-directional vision, structured light, catadioptric camera

\section{Introduction}

In recent decades, multi-directional vision has been increasingly applied in the fields of robot navigation, environment surveillance, etc. due to its advantages of providing rich, high-frequency information at low cost. Compared with traditional vision with a narrow field of view (FOV), which is inadequate for certain applications, the most prominent merit of multi-directional vision is its flexible FOV, therein providing differen$\mathrm{t}$ views of a landscape, which is necessary under some conditions. For example, for robot navigation, if a traditional vision method is employed, then the viewed scene appears discontinuously in the camera within a short period such that it is difficult to avoid an obstacle

\footnotetext{
* Corresponding author. E-mail address:jingxu@tsinghua.edu.cn (J. $\mathrm{Xu})$
}

Preprint submitted to Optics and Lasers in Engineering in a complex unstructured environment with only one camera. In contrast, when multi-directional vision is introduced, scene from different viewpoints is provided such that the robot navigation can be achieved in real time in a narrow space or under partial occlusion [1].

Recently, many attempts have been made to achieve multi-directional vision. One solution is to use multiple cameras to cover an multi-directional scene simultaneously; however, the disadvantages are the cumbersome calibration and alignment of different cameras [2]. An alternative method is to rotate the camera to obtain the multi-directional images by merging sets of images at different viewpoints; however, the disadvantage here is the time-consuming image acquisition process [3]. Another strategy is to use a fisheye lens to capture an multidirectional image, although this suffers from the disadvantage of large distortion [4]. The most used approach

18 January 2017 
is to use a perspective lens and a mirror to capture an multi-directional image in real time. The mirrors usually involve cone, hyperbola, parabola, sphere, or pyramid mirrors[5]. Among these mirrors, a pyramid mirror is a promising method because of its low optical aberration, low manufacturing cost, and uniform imaging resolution[6,7]. Pyramid-mirror-based multi-directional vision was first developed by Nalwa [8-10]. Then, a single pyramid mirror multi-directional vision was extended to a dual mirror pyramid multi-directional vision [11].

Traditional stereo vision obtains 3D profiles using a triangulation of multi-view images with disparity[12]. The largest challenge facing stereo vision is correspondence matching, which is susceptible to scene properties such as object texture, object reflectance, and environmental illumination. To improve the perception reliability and accuracy, artificial structured light (SL) is shot onto the observed object to overcome the lack of texture, leading to a correct correspondence matching regardless of a variant environment. The vision based on SL has certain advantages such as high resolution, high accuracy, high speed, and simple structure, as well as high reliability. Therefore, this technique is increasingly applied to short-distance target sensing with multidirectional vision.

Several 3D multi-directional vision techniques based on SL have been proposed. Four linear laser diodes are employed to form an optical plane around the sensor, which intersects with the observed object, while a catadioptric camera captures the ambient scene with a distorted laser line to obtain the coordinates [13,14]. Similarly, a single laser diode installed with a conical mirror is used to generate an optical plane instead of multiple laser diodes [15]. Additionally, a similar 3D multi-directional measurement system was reported in $[16,17]$. However, these mentioned systems can only measure a slice of a 3D scene in one optical plane of laser incidence. The low spatial resolution of the measured point cloud fails to obtain the entire and detailed profile of the target object.

To improve the spatial resolution, a rotating line laser is used to scan the environment [18]. A Gaussian noise pattern is also projected for region of interest (ROI) reconstruction using stereo multi-directional vision[19]. A similar system has also been proposed, wherein the position and orientation of the synthetic pattern emitter with respect to the camera is flexible and arbitrary for use in a wearable personal assistance system [20]. Four laser diodes with diffractive of elements (DOE) are used to shoot regular dot matrix patterns to obtain a $3 \mathrm{D}$ reconstruction [21]. Similarly, multiple sets of projectors have been used to shoot different encoded patterns onto an ambient scene [23], and then, a catadioptric camera is used to capture the distorted patterns to greatly improve the spatial resolution of the measured point cloud. However, for the above systems, some blind spots still exist when local region reconstruction is performed.

To realize multi-directional reconstruction resolution, a 3D laser-based multi-directional sensor has been developed to obtain a high resolution; however, the mechanical structure is complicated and slow[22]. A fast 3D multi-directional sensor was developed based on a balance of accuracy and resolution in our previous study [24]. The system contains an LED projector and a camera. The encoded pattern of the projector is projected onto the surrounding scene through a hyperbolic mirror; then, the camera installed with a hyperbolic mirror captures the distorted patterns to quickly obtain the 3D point cloud. A similar setup has two parabolic mirrors assembled back-to-back with a camera and a projector [25]. However, the inevitable optical aberration property of curved mirrors will result in an inaccurate $3 \mathrm{D}$ reconstruction. In recent years, the development of a low-cost, high-resolution, and high-accuracy 3D multi-directional sensor that can offer a real-time 3D multi-directional scene has become one of the most popular research issues.

To overcome the above limitations, we presents a 3D multi-directional sensor with high resolution and high accuracy in this paper. Two pyramid mirrors, one for pattern projection and the other one for image acquisition, are introduced to change viewing direction so that the proposed sensor can build the surrounding 3D scene from different viewpoints. There is no optical aberration phenomena in the proposed sensor by using primary mirrors as well. More importantly, it can achieve real-time reconstruction of the scene.

The remainder of this paper is structured as follows: section II analyzes the FOV of the proposed 3D multidirectional sensor; section III depicts the pattern design; section IV gives the calibration method; the mechanical design and sensor performance verification are discussed in section V; and section VI concludes our work.

\section{FOV analysis and optical sensor alignment}

For multi-directional vision based on a pyramid mirror, each face of the pyramid mirror will reflect part of the scene into an optical sensor (e.g., a camera) to form an undistorted wide-FOV multi-directional image with singe or multiple viewpoints. The advantage of a single viewpoint is the ability to generate a seamless multi- 
directional image at arbitrary distances. However, the single viewpoint has to satisfy the strict geometric constraint and is difficult to align. In contrast, the multiple viewpoints can be easily realized by a single pyramid mirror with a compact structure. To simplify the structure, a single pyramid mirror with multiple viewpoints is employed in this paper. To maximize the FOV of multiple viewpoints, we establish the mathematic model of the FOV in terms of the optical sensor and the pyramid mirror.

Without loss of generality, the base of a regular pyramid mirror is assumed to be square, and the pyramid mirror and optical sensor are well aligned. The dimensions and layout of the pyramid mirror and the optical sensor, as shown in Fig. 1, are assumed to be as follows. The base of the pyramid mirror is on the $x-y$ plane of the pyramid mirror coordinate system, and the length of the base edge is set to be $2 a$. The slant angle between the square base and the triangle face is $\theta$. In this case, the four vertices of the rectangle base are located on the $\mathrm{x}$-axis and $\mathrm{y}$-axis, with coordinates of $(\sqrt{2} a, 0,0)$, $(0, \sqrt{2} a, 0),(-\sqrt{2} a, 0,0)$, and $(0,-\sqrt{2} a, 0)$, whereas the pyramid mirror apex is located at $(0,0, a \tan \theta)$ in the pyramid mirror coordinate system. The optical center of the optical sensor is located on the z-axis a distance of $H$ from the pyramid mirror apex; thus, the optical center $O_{c}$ of the optical sensor is located at $(0,0, a \tan \theta+H)$ in the pyramid mirror coordinate system. The width and height of the optical sensor chip are $2 u$ and $2 v$, and the center of the optical sensor chip is located on the z-axis of the pyramid mirror coordinate system. The focal length of the lens of the optical sensor is $f$, and the xyz-axes of the camera coordinate system are supposed to be parallel to those of the pyramid mirror coordinate system.

\subsection{FOV analysis}

When the optical sensor chip size and pyramid mirror dimensions are assigned, the relationship between the FOV of the multi-directional sensor and the optical sensor location $H$ and the focal length $f$ of the lens are investigated to maximize the FOV.

Due to the symmetrical distribution of the four triangle faces of the pyramid mirror, only the first quadrant of the pyramid mirror is analyzed.

Concerning the reflection property of the planar mirror, the reflected light ray through the optical center $O_{c}$ of the optical sensor can be determined by the incoming light ray through the virtual optical center $O_{c}^{\prime}$. There-

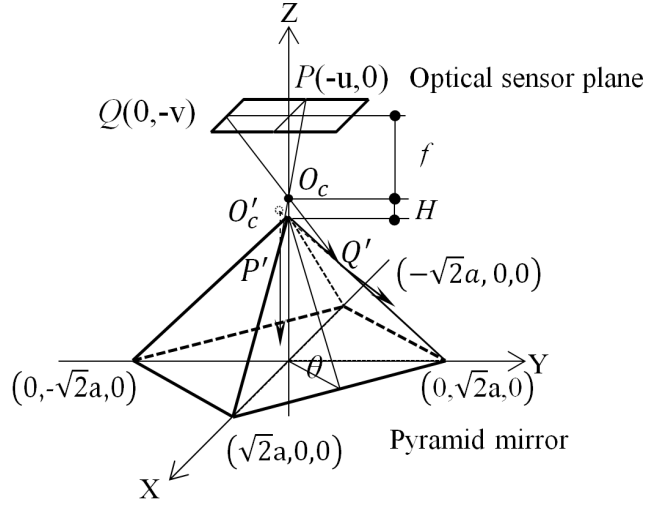

Fig. 1. Dimensions and layout of the pyramid mirror and the optical sensor: the four vertices of the rectangle base of the pyramid mirror are $(\sqrt{2} a, 0,0),(0, \sqrt{2} a, 0),(-\sqrt{2} a, 0,0)$, and $(0,-\sqrt{2} a, 0)$; the apex of the pyramid mirror is at $(0,0, a \tan \theta)$; the optical center of the optical sensor is on the $\mathrm{z}$-axis a distance $H$ from the pyramid mirror apex; the focal length of the lens is $f$; and the coordinate systems of the pyramid mirror and the optical sensor are parallel.

fore, the location of the virtual optical center $O_{c}^{\prime}$ has to be determined to analyze the FOV.

Three vertices of the triangle face of the first quadrant, as shown in Fig. 1, are at $(\sqrt{2} a, 0,0),(0, \sqrt{2} a, 0)$, and $(0,0, a \tan \theta)$. Then, the equation for the plane of the triangle face of the first quadrant is

$$
\frac{\sqrt{2}}{2} \sin \theta \cdot x+\frac{\sqrt{2}}{2} \sin \theta \cdot y+\cos \theta \cdot z-a \sin \theta=0
$$

where $x>0, y>0$.

Thus, the virtual optical center of camera $O_{c}^{\prime}$ in the pyramid mirror coordinate system is

$$
\left(-\frac{\sqrt{2}}{2} H \sin (2 \theta),-\frac{\sqrt{2}}{2} H \sin (2 \theta), a \tan \theta+H-2 H \cos ^{2} \theta\right) \text {. }
$$

The imaging region of the optical sensor chip for the triangle face of the first quadrant is a rectangle with four vertices $(0,0),(-u, 0),(-u,-v)$, and $(0,-v)$ on the image plane; then, the corresponding viewing vector for four pixels on rectangle vertices are $(0,0,-f)$, $(u, 0,-f),(u, v,-f)$, and $(0, v,-f)$ in the pyramid mirror coordinate system.

Thus, the intersection points $P^{\prime}$ and $Q^{\prime}$, as shown in Fig. 1, between the limit view vectors from pixels $P$ and $Q$ and the edges of the triangle face are $\left(\frac{H \frac{u}{f}}{1-\frac{\sqrt{2}}{2} \frac{u}{f} \tan \theta}, 0, H+a \tan \theta-\frac{H}{1-\frac{\sqrt{2}}{2} \frac{u}{f} \tan \theta}\right)$,
$\left(0, \frac{H \frac{v}{f}}{1-\frac{\sqrt{2}}{2} \frac{v}{f} \tan \theta}, H+a \tan \theta-\frac{H}{1-\frac{\sqrt{2}}{2} \frac{v}{f} \tan \theta}\right)$.

Therefore, the viewing vector $O_{c}^{\prime} P^{\prime}$ from the virtual focal center is

$$
\left(\frac{\frac{\sqrt{2}}{2} H \sin 2 \theta+H \frac{u}{f} \cos ^{2} \theta}{1-\frac{\sqrt{2}}{2} \frac{u}{f} \tan \theta}, \frac{\sqrt{2}}{2} H \sin 2 \theta, 2 H \cos ^{2} \theta-\frac{H}{1-\frac{\sqrt{2}}{2} \frac{u}{f} \tan \theta}\right)
$$




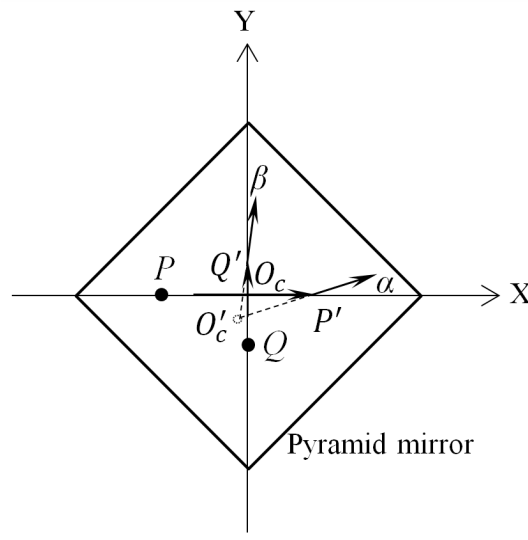

Fig. 2. FOV on the $x-y$ plane

Similarly, the viewing vector $O_{c}^{\prime} Q^{\prime}$ from the virtual focal center is

$\left(\frac{\sqrt{2}}{2} H \sin 2 \theta, \frac{\frac{\sqrt{2}}{2} H \sin 2 \theta+H \frac{v}{f} \cos ^{2} \theta}{1-\frac{\sqrt{2}}{2} \frac{v}{f} \tan \theta}, 2 H \cos ^{2} \theta-\frac{H}{1-\frac{\sqrt{2}}{2} \frac{v}{f} \tan \theta}\right)$

Therefore, we have the angle $\alpha$ from the $\mathrm{x}$-axis to the xy components of the viewing vectors $O^{\prime}{ }_{c} P^{\prime}$, as shown in Fig. 2:

$$
\alpha=a \tan \left(\frac{\sqrt{2} \tan \theta-\frac{u}{f} \tan ^{2} \theta}{\sqrt{2} \tan \theta+\frac{u}{f}}\right)
$$

Similarly, we have the angle $\beta$ from the xy components of the viewing vector $O_{c}^{\prime} P^{\prime}$ to the y-axis

$$
\beta=a \tan \left(\frac{\sqrt{2} \tan \theta-\frac{v}{f} \tan ^{2} \theta}{\sqrt{2} \tan \theta+\frac{v}{f}}\right)
$$

The horizontal FOV in the xy-plane for the first quadrant is

$$
\frac{\pi}{2}-(\alpha+\beta)
$$

where

$$
\alpha+\beta=\operatorname{atan}\left(\frac{\frac{\sin ^{2}(2 \theta)}{\cos (2 \theta)}\left(2-\frac{u v}{f^{2}}\right)+\sqrt{2} \sin (2 \theta) \frac{u+v}{f}}{\frac{2 u v}{f^{2}}+\sqrt{2} \tan (2 \theta) \frac{u+v}{f}}\right)
$$

Maximizing the horizontal FOV is equivalent to minimizing Eq. (5).

From Eq. (5), we know that the horizontal FOV is constant, regardless of the optical sensor location $H$.

Moreover, to maximize the FOV, $(\alpha+\beta)=0$ is preferred, i.e., the optimal focal length $f$ of the lens is

$$
f=\frac{\sqrt{(u+v)^{2}+4 u v \tan ^{2}(2 \theta)}-(u+v)}{2 \sqrt{2} \tan (2 \theta)}
$$

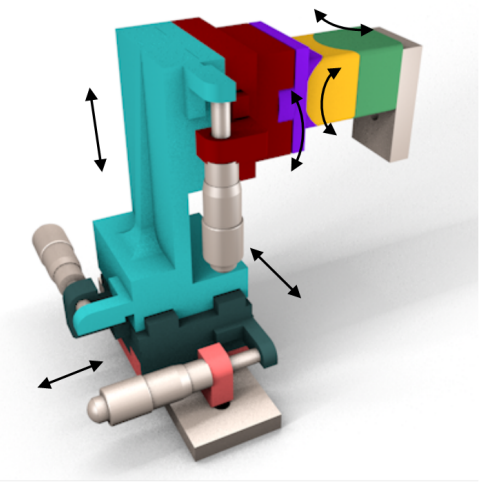

Fig. 3. 6-DOF alignment stage

\subsection{Optical sensor alignment}

The real FOV is determined by the optical sensor's intrinsic and extrinsic parameters with respect to the pyramid mirror.

To align the camera, the camera is calibrated in advanced such that the optical center location with respect to the camera is known. The precise machining of the connection structure makes the optical center quite close to the desired location. The desired captured image of the markers distributed in the environment can be calculated when the camera is well aligned. The camera is finely adjusted until the real captured image approximately approaches the desired image.

Compared with the camera alignment, the projector alignment is significantly more difficult because the projector is not capable of viewing the scene. Thus, we use a 6-DOF (degree-of-freedom) alignment stage, as shown in Fig. 3, providing precise translation along the $\mathrm{x}$-axis, $\mathrm{y}$-axis, and $\mathrm{z}$-axis and precise rotation about the $\mathrm{x}$-axis, $\mathrm{y}$-axis, and $\mathrm{z}$-axis. Assuming that the projector is aligned, we can obtain the desired illumination of the scene with a designed pattern, e.g., a chessboard pattern. Using the difference between the desired and real illuminations, we can adjust the projector with the 6DOF alignment stage until alignment is achieved.

\section{Pattern design}

To determine the correspondence between the camera and the projector, a series of phase-shifting fringe patterns are shot from the projector and imaged into the camera. The $n^{\text {th }}$ shot pattern $I_{n}$ can be represented by[26]

$$
I_{n}(x, y)=I^{\prime}+I^{\prime \prime} \cos \left(\phi(x, y)-\frac{2 \pi n}{N}\right)
$$


where $I^{\prime}$ is the average intensity, $I^{\prime \prime}$ is the amplitude, $\phi(x, y)$ is the phase, $N$ is the total number of shot patterns, and $n$ is from 0 to $N-1$.

The phase $\phi(x, y)$ can be obtained by

$$
\phi(x, y)=\tan ^{-1}\left(\frac{\sum_{n=0}^{N-1} I_{n}(x, y) \sin \left(\frac{2 \pi n}{N}\right)}{\sum_{n=0}^{N-1} I_{n}(x, y) \cos \left(\frac{2 \pi n}{N}\right)}\right)
$$

To achieve real-time measurements, the minimum number of fringe patterns is three [27]:

$$
\left\{\begin{array}{c}
I_{0}(x, y)=I^{\prime}+I^{\prime \prime} \cos \left(\phi(x, y)-\frac{2 \pi}{3}\right) \\
I_{1}(x, y)=I^{\prime}+I^{\prime \prime} \cos (\phi(x, y)) \\
I_{2}(x, y)=I^{\prime}+I^{\prime \prime} \cos \left(\phi(x, y)+\frac{2 \pi}{3}\right)
\end{array}\right.
$$

For the three-step phase-shifting fringe pattern, the phase $\phi(x, y)$ can be obtained by

$$
\phi(x, y)=\tan ^{-1}\left(\frac{\sqrt{3}\left(I_{0}(x, y)-I_{2}(x, y)\right)}{2 I_{1}(x, y)-I_{0}(x, y)-I_{2}(x, y)}\right)
$$

It is noted that the obtained relative periodic phase is from $-\pi$ to $\pi$. To remove any ambiguity, the fringe order has to be specified to obtain the absolute phase. To this end, a dual-frequency, phase-shifting fringe pattern is introduced in this paper [28,29]. Using the lowfrequency phase $\phi_{l}$ and high-frequency phase $\phi_{h}$ at the same pixel $(x, y)$, we can obtain the fringe order $W$ :

$$
W=\left[\frac{L \phi_{l}-\phi_{h}}{2 \pi}\right]
$$

where $L=\frac{f_{h}}{f_{l}}$, [] is the nearest rounding operator.

As long as the fringe order $W$ is obtained, the absolute phase $\phi_{s}$ can be achieved:

$$
\phi_{s}=W \cdot 2 \pi+\phi_{h}
$$

\section{Calibration}

A critical step for obtaining accurate measurements is to well calibrate the sensor. The calibration procedure involves virtual camera-projector calibration for each triangle face of the pyramid mirror and sensor calibration to integrate the four quadrants of the triangle faces into a uniform one. This section depicts how the sensor is well calibrated.

\subsection{Quadrant camera-projector calibration}

The quadrant camera-projector calibration consists of specifying the geometric parameter between the virtual

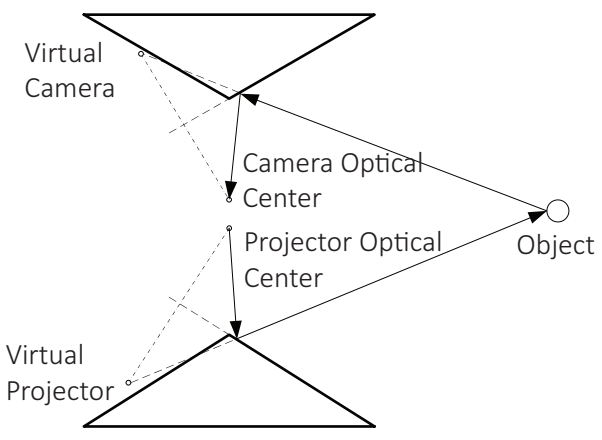

Fig. 4. Virtual camera-projector calibration

projector and the virtual camera due to the reflection of the pyramid mirror, as shown in Fig. 4. In this paper, we adopt a planar circle calibration gauge, where the world coordinate system is established on the planar gauge and $Z_{w}=0$ [30]. The mathematic model of the virtual camera is described as follows:

$$
s\left[\begin{array}{c}
u_{c} \\
v_{c} \\
1
\end{array}\right]=K_{c}\left[\begin{array}{lll}
r_{1} & r_{2} & t
\end{array}\right]\left[\begin{array}{c}
X_{w} \\
Y_{w} \\
1
\end{array}\right]
$$

where $s$ is a scale factor, $\left[\begin{array}{lll}u_{c} & v_{c} & 1\end{array}\right]^{T}$ is the image coordinate of the circle center; $K_{c}$ is the camera intrinsic parameter; $\left[\begin{array}{ll}r_{1} & r_{2}\end{array}\right]$ is the camera extrinsic parameter; $\left[\begin{array}{lll}X_{w} & Y_{w} & 1\end{array}\right]^{T}$ is the world coordinate of the circle center.

In this technique, an accurate extraction of the circle center is a prerequisite for precise calibration. To this end, sub-pixel edge detection and ellipse fitting algorithms are introduced.

Prior to edge detection, the preprocessing algorithm$\mathrm{s}$, including noise filtering and threshold segmentation, are applied to the original image first. The noise filtering consists of a Gaussian filter that maintains edge features and removes noise. Then, a Canny operator is used to detect the edge of the circle. After that, the edge boundary is used to fit an ellipse to precisely locate the ellipse center.

For the virtual projector, the mathematic model is also described as

$$
s\left[\begin{array}{c}
u_{p} \\
v_{p} \\
1
\end{array}\right]=K_{p}\left[\begin{array}{lll}
r_{1}^{\prime} & r_{2}^{\prime} & t^{\prime}
\end{array}\right]\left[\begin{array}{c}
X_{w} \\
Y_{w} \\
1
\end{array}\right]
$$

The biggest challenge for the projector calibration is the lack of an ability to observe the scene; thus, we use 
phase shifting to establish the pixel location on the projector image plane and the circle center on the calibration gauge. To improve the accuracy of phase shifting, it is better to adopt more steps in the phase shifting.

For each couple of virtual camera-projector calibrations, the coordinate system is established on the optical center of the virtual camera. The transformation matrix from the virtual projector to the virtual camera in a camera-projector pair can be estimated by ${ }^{V P} T_{V C}=$ $\left[r_{1}, r_{2}, t\right] \times\left[r_{1}^{\prime}, r_{2}^{\prime}, t^{\prime}\right]^{T}$.

\subsection{Sensor calibration}

The sensor calibration attempts to integrate four virtual camera-projector pairs into a uniform coordinate system, resulting in an entire reconstructed point cloud. Therefore, the position and orientation of each triangle face needs to be calibrated. To this end, the Householder reflection was applied. Every triangle face can be expressed by $\{m, l\}$ as shown in Fig. 1, where $m$ denotes the unit normal vector of the mirror plane and $|l|$ indicates the distance of the origin to the mirror plane. For the triangle face of the first quadrant, $m_{1}=$ $\left[\frac{\sqrt{2}}{2} \sin \theta, \frac{\sqrt{2}}{2} \sin \theta, \cos \theta\right]^{T}$ and $l_{1}=-a \sin \theta$, so that the pose (position and orientation) of the first mirrored virtual camera can be estimated by:

${ }^{V C 1} T_{B}=\left[\begin{array}{cc}I-2 e e e^{T} & 0_{3 \times 1} \\ 01 \times 3 & 1\end{array}\right]\left[\begin{array}{cc}I-2 m_{1} m_{1}^{T} & 2 l_{1} m_{1} \\ 01 \times 3 & 1\end{array}\right]{ }^{C} T_{B}={ }^{V C 1} H_{C} \cdot{ }^{C} T_{B}$

where ${ }^{B} T_{C}$ represent the pose of the camera with reference to the base coordinate system, $e$ can be $[1,0,0]^{T}$, $[0,1,0]^{T}$ or $[0,0,1]^{T}$, implying the reflection axis and making the left-handed coordinate system right-handed. Similarly, the pose of the second virtual camera reflected by the triangle face of the second quadrant can be estimated by:

${ }^{V C 2} T_{B}=\left[\begin{array}{cc}I-2 e e^{T} & 0_{3 \times 1} \\ 01 \times 3 & 1\end{array}\right]\left[\begin{array}{cc}I-2 m_{2} m_{2}^{T} & 2 l_{2} m_{2} \\ 01 \times 3 & 1\end{array}\right]{ }^{C} T_{B}={ }^{V C 2} H_{C} \cdot{ }^{C} T_{B}$

Therefore the transformation matrix between these cameras is express by:

$$
{ }^{V C 2} H_{V C 1}={ }^{V C 2} H_{C}\left({ }^{V C 1} H_{C}\right)^{-1}
$$

It is shown from the above equations that the transformation matrices between virtual cameras are only relevant to the dimension of the pyramid mirror. Since the base edge $2 a$ and the slant angle $\theta$ of the pyramid mirror are known, we can calculate the transformation matrices and integrate four pairs of virtual cameras into one coordinate system, that is, the reconstructed point cloud can be merged into an entire one.

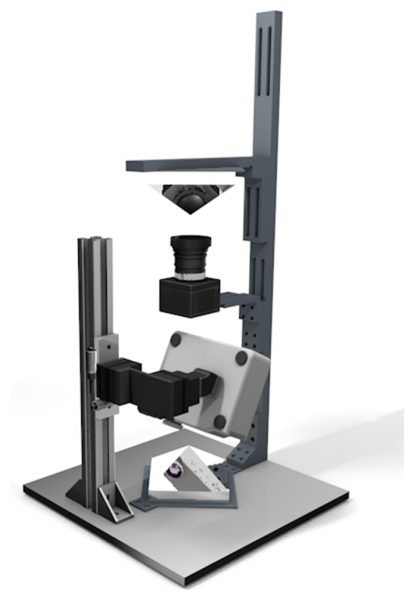

Fig. 5. Configuration of the 3D multi-directional sensor

\section{Experimental verification}

The proposed 3D multi-directional sensor, as shown in Fig. 5, consists of a monochromatic camera with a resolution of $1280 \times 1024$ (Manufacturer: DaHeng; Model: DH-HV1351UM), a DLP projector with a resolution of $800 \times 600$ (Manufacturer: LG; Model: HS200), two pyramid mirrors, some mechanical structures, and connections.

It is emphasized that, when the camera aperture (determining the conic angle of a bundle of light rays passing through a focus on the image plane) is narrow, only highly collimated rays are accepted, leading to sharp images; on the other hand, when the camera aperture is wide, uncollimated rays are accepted, resulting in blurred images, especially for the edge region of a triangle face, which absorbs rays coming from different triangle faces. In addition, similar to a camera, if the projector aperture is not sufficiently narrow, the shot pattern on the edge region of the triangle face will be blurred, that is, the light rays from the same pixel in the projector would go to two adjacent triangle faces simultaneously. To solve this problem, the intensity of the shot pattern near the edge region of the triangle face is set 0 .

\subsection{Calibration}

To calibrate the virtual camera-projector, a planar circle calibration gauge is used, as shown in Fig. 6, where the five larger dots determine the $\mathrm{x}$-axis and $\mathrm{y}$-axis of the calibration gauge; the distance between adjacent dots is $25 \mathrm{~mm}$ along the $\mathrm{x}$-axis and $\mathrm{y}$-axis. The calibration errors for the camera are 0.084 pixels and 0.076 pixel- 
$\mathrm{s}$ in the $\mathrm{x}$-axis and $\mathrm{y}$-axis of image coordinate frame, respectively, whereas the calibration errors for the projector are 0.228 pixels and 0.160 pixels, respectively.

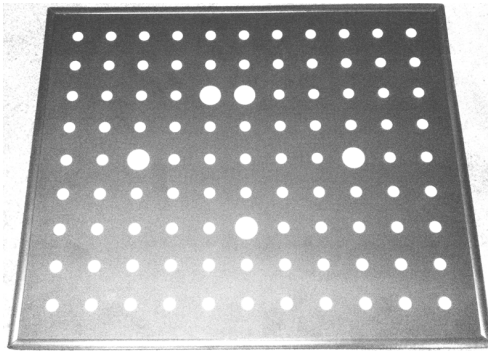

Fig. 6. Calibration gauge of each virtual projector-camera pair

To calibrate the sensor, the dimensions of the pyramid mirror are precisely measured with $2 a=100.10 \mathrm{~mm}$, $\theta=33.63^{\circ}$. By applying the sensor calibration, four groups of measured point clouds are merged into a $\mathrm{u}-$ niform coordinate system. As described in Section 4.2, transformation matrix between two adjacent cameraprojector pairs depends only on the slant angle $\theta$ and length $a$. However, $\theta$ is obtained by the height $h$ of the mirror pyramid during designing and manufacturing and should be calculated by:

$$
\theta=\arctan (h / a)
$$

In order to verify the reliability of the sensor calibration method, we simulated the influence under height error $\delta h$ and edge error $\delta a$. The calibration result here will be split into a Rodriguez vector $\mu$ for rotation and a translation vector $v$, whose norms representing the rotational angle error $\|\Delta \mu\|$ and translational error $\|\Delta v\|$ respectively. The simulation results are shown in Fig. 7 and 8 with $a=50 \mathrm{~mm}$ and $h=25 \mathrm{~mm}$. One observation is that $\|\Delta \mu\|$ and $\|\Delta v\|$ are still small even though errors of $0.1 \mathrm{~mm}$, which can be easily achieved in manufacturing, exist for both $a$ and $h$. The simulation results show that the manufacturing accuracy of the mirror is sufficiently reliable to calibrate the sensor without calibrating the mirror orientations.

\subsection{Numerical simulation of misalignment}

To analyze the influence of misalignment on the FOV, a numerical simulation is used in this paper. The translation misalignment along the $\mathrm{x}$-axis and $\mathrm{y}$-axis is equivalent to small errors of $u$ and $v$, and the translation misalignment along the z-axis has an influence on the FOV. Moreover, the rotation misalignment about the $\mathrm{x}$-axis and y-axis is symmetrical; therefore, we only analyze the rotation misalignment about the $\mathrm{x}$-axis and $\mathrm{z}$-axis.

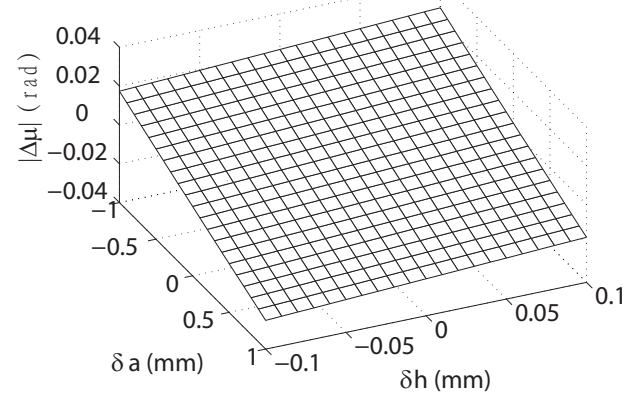

Fig. 7. Simulation result of rotation angle error

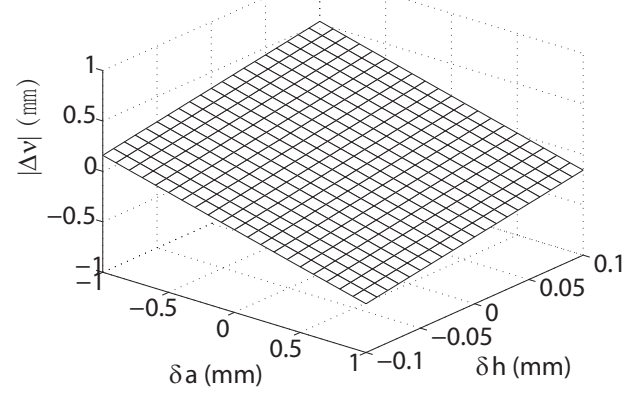

Fig. 8. Simulation result of translation error

The parameters are set as follows: $u=640, v=512$, $f=700$, and $\theta=35^{\circ}$. The results are displayed in Fig. 9 and Fig. 10.

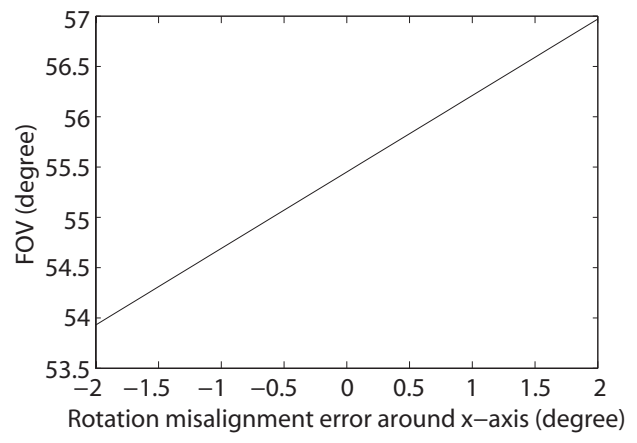

Fig. 9. Relationship between FOV and rotation misalignment error about the $\mathrm{x}$-axis

The results show that the FOV is monotonically increased with respect to the rotation misalignment error about the $\mathrm{x}$-axis, whereas the FOV is symmetrically increased with respect to the magnitude of the rotation misalignment error about the $\mathrm{x}$-axis.

\section{3. $3 D$ measurement}

To verify the measurement accuracy and feasibility of the proposed 3D multi-directional sensor, four exper- 


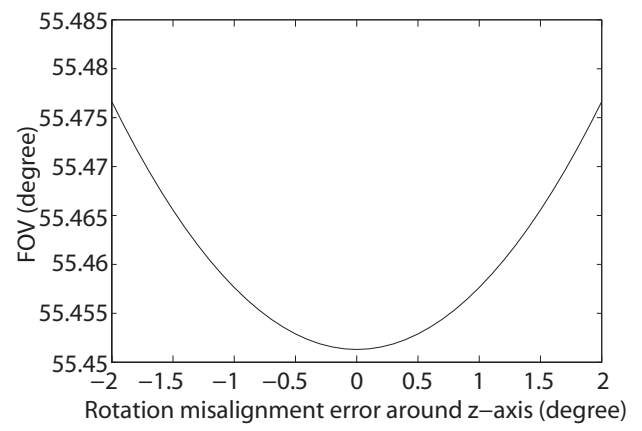

Fig. 10. Relationship between FOV and rotation misalignment error about the z-axis

iments are performed as follows.

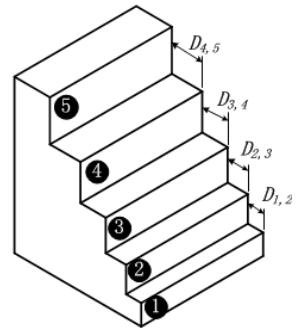

(a)

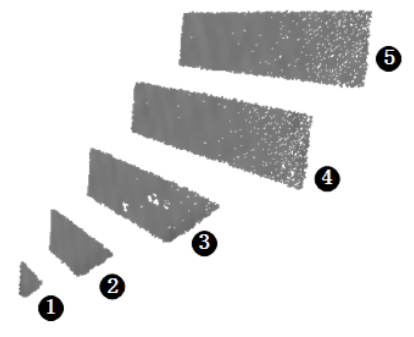

(b)
Fig. 11. 3D measurement for five-step block: (a) five-step block; (b) measured point cloud

In the first experiment, a five-step block is used to validate the measurement accuracy of a plane, as shown in 11(a). The measured points of the five planes, as shown in 11(b), are assumed to be parallel with each other. The plane can be represented by

$$
n_{x} x+n_{y} y+n_{z} z+d_{i}=0
$$

where $\left[\begin{array}{lll}n_{x} & n_{y} & n_{z}\end{array}\right]$ is the plane normal vector yielding $\sqrt{n_{x}^{2} x+n_{y}^{2} y+n_{z}^{2}}=1$, and $d_{i}$ is the distance from the origin to the plane $i(i=1,2, \ldots 5)$.

The five measured point clouds are used to fit the five parallel planes after denoising. The mean value and root mean square (RMS) of the distances between points and fitted planes are shown in Table 1, which shows that the plane fitting error is approximately $0.25 \mathrm{~mm}$.

The measurement error between planes is defined by the distance between two adjacent planes $i$ and $i+1$ as

$$
D_{i, i+1}=\left|d_{i}-d_{i+1}\right|
$$

where $i(i=1,2, \ldots 4)$. The measurement error is shown in Table 2, where the real value is obtained using a laser radar (Model: MV330, Manufacture: Nikon). The result shows that the measurement error between planes is less than $1 \mathrm{~mm}$.
Table 1

The plane fitting error.

\begin{tabular}{ccc}
\hline Plane & $\begin{array}{c}\text { Mean } \\
\text { value(mm) }\end{array}$ & $\begin{array}{c}\text { RMS } \\
\text { value(mm) }\end{array}$ \\
\hline 1 & 0.159 & 0.204 \\
2 & 0.192 & 0.252 \\
3 & 0.186 & 0.243 \\
4 & 0.184 & 0.236 \\
5 & 0.202 & 0.258 \\
\hline
\end{tabular}

Table 2

The measurement error between planes.

\begin{tabular}{cccc}
\hline Distance & Real & Measured & \\
& Error $(\mathrm{mm})$ \\
& value(mm) value(mm) & \\
\hline$D_{1,2}$ & 15.039 & 14.163 & 0.876 \\
$D_{2,3}$ & 19.844 & 19.525 & 0.319 \\
$D_{3,4}$ & 25.157 & 24.652 & 0.505 \\
$D_{4,5}$ & 30.180 & 30.844 & 0.664 \\
\hline
\end{tabular}

In the second experiment, a cone is used to evaluate the measurement accuracy for a curved surface. The measured point cloud and the fitted conical surface are shown in Fig. 12(a) and 12(b). The distance between a point and the conical surface is regarded as the measurement error. The experimental result shows that the RMS of the measurement error for a conical surface is $0.491 \mathrm{~mm}$.

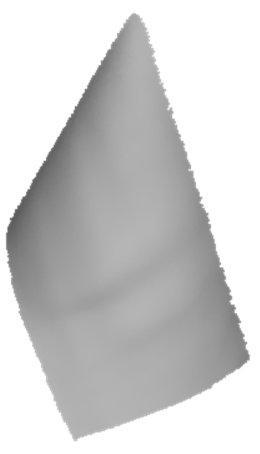

(a)

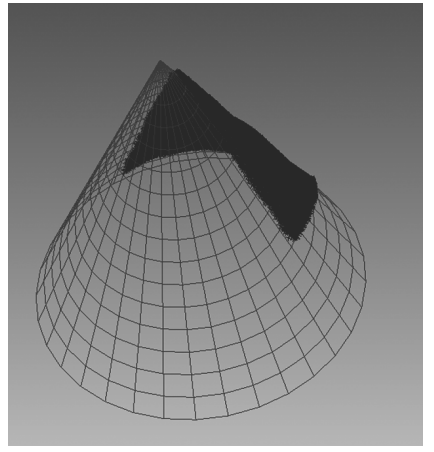

(b)
Fig. 12. 3D measurement for a conical surface: (a) measured point cloud; (b) fitted conical surface

In the third experiment, two perpendicular planes (as shown in Fig. 13(a)) was reconstructed and the included angle was estimated in order to validate the sensor calibration. For the purpose of comparison, two printed circle calibration gauge with a grid size of $20 \mathrm{~mm} \times 20 \mathrm{~mm}$ were pasted on the planes respectively. The circle centers are extracted and their 3D coordinates are calcu- 
lated using monocular method with a GO-5000M-USB JAI camera as shown in Fig. 13(b). The normal vectors of the fitted planes were used to calculate the angle, $1.516 \mathrm{rad}$ as the result. Afterwards, the proposed sensor was utilized to obtain point clouds of the planes (as shown in Fig. 13(c) and Fig. 13(d)) and the measured value of the angle was $1.488 \mathrm{rad}$. The difference is less than $0.03 \mathrm{rad}$, verifying accuracy of the sensor calibration.

Finally, four plaster statues with complex shapes are placed around the developed sensor to test its performance. The projector emits a pattern to the scene through the pyramid mirror, while the camera acquires the deformed pattern through the pyramid mirror. Then, the correspondence between the virtual camera and the virtual projector is established, and a $3 \mathrm{D}$ point cloud is reconstructed via a triangulation technique. It is noted that the high-frequency phase-shifting fringe is used to determine the accurate relative phase. In contrast, the low-frequency phase-shifting fringe exhibits a smaller amplitude with low noise ration; therefore, it is used to specify the fringe order. For each virtual cameraprojector pair, the corresponding point cloud is reconstructed. Subsequently, four groups of point clouds are integrated via sensor calibration. The four plaster statues and corresponding point clouds are shown in Fig. 14(a) and Fig. 14(b). One of the local enlarged plaster statues and the corresponding point cloud are shown in Fig. 14(c) and Fig. 14(d). The experimental results show that the proposed 3D multi-directional sensor is feasible and that it achieves good measurement accuracy.

\section{Conclusion}

This paper attempts to design a 3D, high-resolution, high-accuracy multi-directional sensor by using pyramid mirrors. The FOV of the proposed 3D multidirectional sensor is analyzed, and the mathematical model is given. The dual-frequency phase-shifting fringe is used for real-time measurements. The planar circle calibration gauge and the Householder reflection constraint are adopted to calibrate the 3D multidirectional sensor. A 3D multi-directional sensor prototype is developed and constructed using the key components of a catadioptrical camera, a DLP projector, and two pyramid mirrors. Experiments were performed and analyzed to verify the accuracy and feasibility of the proposed 3D multi-directional sensor.

The significant advantages of the proposed sensor include (1) Two planar pyramid mirrors instead of tradi-

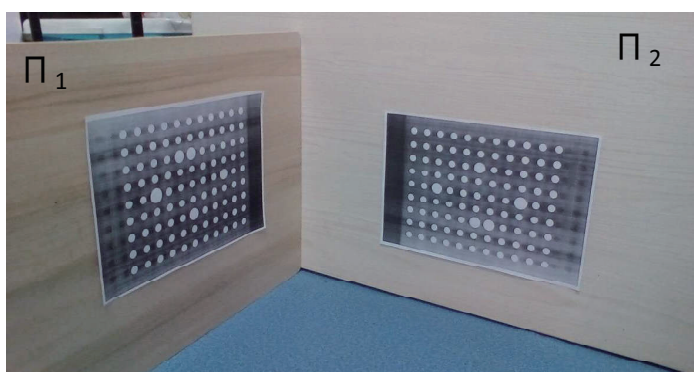

(a)

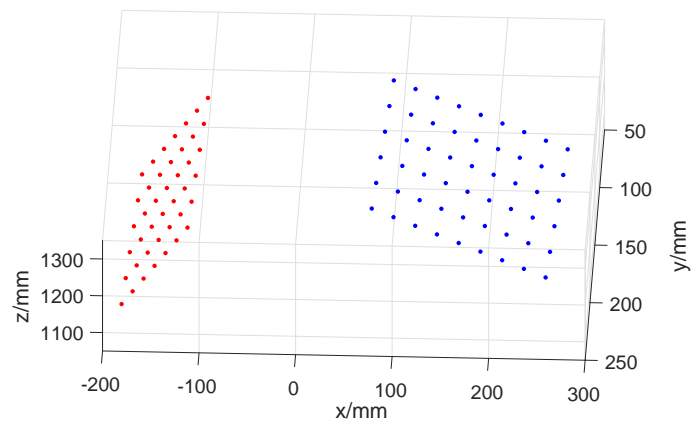

(b)

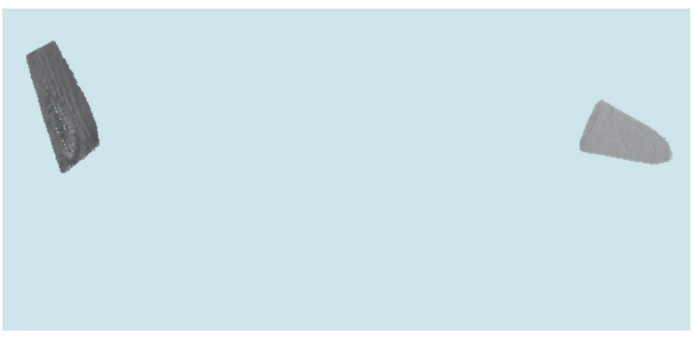

(c)

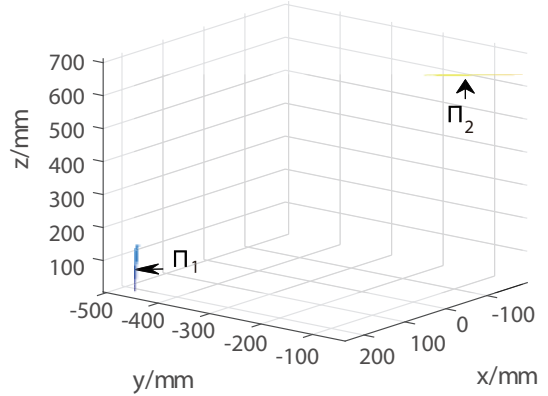

(d)

Fig. 13. Validation for sensor calibration: (a) two perpendicular planes to be measured; (b) circle centers estimated by monocular method; (c) reconstructed point clouds; (d) another view of the point clouds 


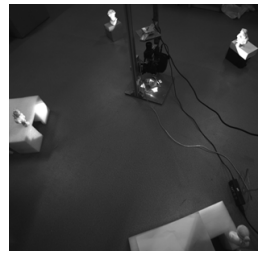

(a)

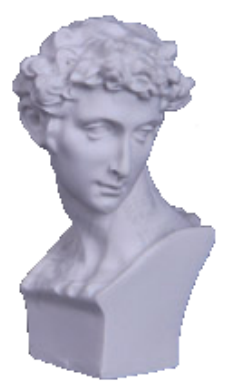

(c)
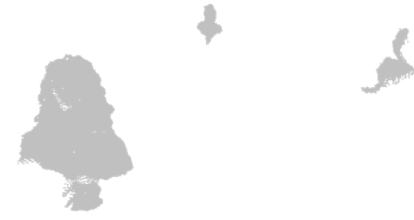

(b)

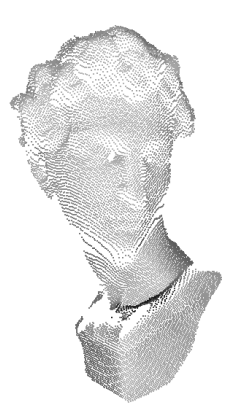

(d)
Fig. 14. 3D measurement for plaster statues with complex shape: (a) four plaster statues; (b) global point cloud; (c) one plaster statue; (d) local enlarged point cloud

tional curved mirrors are used, thereby reducing optical aberration phenomena and resulting in improved reconstruction accuracy; (2) Although similar effect can be achieved with four cameras and four projectors, our proposed sensor uses only one camera and one projector with the assistance of two pyramid mirrors and results in low cost; (3) Furthermore, the calibration procedure should be conducted for several times for multiple cameras and multiple projectors since hardware differences exist, and synchronization should be rigorously followed to achieve real-time performance. But these are not needed for the proposed sensor, making it easyto-use.

Nevertheless, the total FOVs of the camera and the projector are divided into four directions by pyramid mirrors so that FOV in one direction is narrowed and the viewpoints of the camera and the projector are restricted, compared with four-camera-and-four-projector sensors. Blind area appears between the FOVs reflected by two adjacent mirrors. And the structure of the sensor should be further designed to enlarge the vertical FOV.

\section{Acknowledgment}

The work is partially supported by the National Science Foundation of China (No.U1613205 and 51675291), the Fund of the State Key Laboratory of Tribology of China (No. SKLT2015B10), and the Basic
Research Program of Shenzhen(No.JCYJ20160229123030978 and JCYJ20150828104330541).

\section{References}

[1] Rituerto A, Puig L, Guerrero J J. Comparison of omnidirectional andconventional monocular systems for visual SLAM. In: The 10th Workshop on Omnidirectional Vision, Camera Networks and Non-classical Cameras, Zaragoza, Spain, June 27, 2010. p. 1-13.

[2] Firoozfam P. Negahdaripour S. A multi-camera conical imaging system forrobust 3D motion estimation, positioning and mapping from UAVs. In: IEEE Conference onAdvanced Video and Signal Based Surveillance, Miami, USA, July 21-22, 2003. p. 99-106.

[3] Huang H C and Hung Y P. Panoramic stereo imaging system with automatic disparity warping and seaming, Graphical Models and Image Processing, 1998, 60(3): 196-208.

[4] Courbon J, Mezouar Y, Eck L , Martine P. A generic fisheye camera model for robotic applications. Proceedings of the 2007 IEEE/RSJ International Conference on Intelligent Robots and Systems, San Diego, CA, USA, Oct 29 - Nov 2, 2007, pp.16831688

[5] Benosman R. Kang S B. Panoramic Vision: Sensors, Theory, and Applications. New York: Springer, 2001.

[6] Zeng L, Zhang W G, Zhang S P, Wang D W. Video image mosaic implement based on planar-mirror-based catadioptric system. Signal, Image and Video Processing, 8(6), 2014, pp1007-1014.

[7] Tan K H, Hua H, Ahuja N. Multiview panoramic cameras using mirror pyramids. IEEE Transactions on Pattern Analysis and Machine Intelligence, 26(7), 2004, pp941-946.

[8] Nalwa V, A true omnidirectional viewer, Technical Report, Bell Laboratories, 1996

[9] Nalwa V, Compact high-resolution panoramic viewing system, US Patent 6,195,204B1, 2001

[10] Nalwa V, Panoramic viewing system with a composite field of view, US Patent 6,700,711, 2004.

[11] Hua H, Ahuja N, Gao C. Design analysis of a high resolution panoramic camera using conventional imagers and a mirrorpyramid, IEEE Transactions on Pattern Analysis and Machine Intelligence 29(2),2007, pp356C361.

[12] Wang TQ and Chen QJ. Object semantic map representation for indoor mobile robots. In: International Conference on System Science and Engineering, Macao, China, June 8-10, 2011. p. 309-13.

[13] Yi S, Choi B, Ahuja N. Real-time omnidirectional distance measurement withactive panoramic vision. International Journal of Control, Automation, and Systems 2007, 5(2): 184-91.

[14] Yi S, Suh J, Hong Y, Hwang D. Active ranging system based on structured laserlight image. In: Proceedings of SICE Annual Conference, Taipei, China, Auguse 18-21, 2010. p. 747-52.

[15] Matsui K, Yamashita A, Kaneko T. 3-D shape measurement of pipe by range finder constructed with omni-directional laser and omni-directional camera. In: IEEE International Conference on Robotics and Automation, Anchorage, USA, May 3-7, 2010. p. 2537-42.

[16] Orghidan R, Salvi J, Mouaddib E M. Modelling and accuracy estimation of a newomnidirectional depth computation sensor. Pattern Recognition Letters 2006, 27(7): 843-53. 
[17] Zhou F Q, Peng B, Cui Y, Wang Y X, Tan H S. A novel laser vision sensor foromnidirectional 3D measurement. Optics \& Laser Technology 2013, 45: 1-12.

[18] Xu J, Gao B T, Liu C D, Wang P, Gao S L. An omnidirectional 3D sensor with line laser scanning, Optics and Lasers in Engineering, 2016, 84: 96-104

[19] Gledhill D, Yun G, Taylor D, Clarke D. 3D reconstruction of a region of interest using structured light and stereo panoramic images. IEEE Proceedings of the Eighth International Conference on Information Visualisation, 2004, 1007-1012.

[20] Paniagua C, Puig L and J.Guerrero J. Omnidirectional structured light in a flexible configuration. Sensors 2013, 13, 13903-13916.

[21] Jia T, Shi Y, Zhou Z X, Chen D Y. 3D depth information extraction with omnidirectional camera, Information Processing Letters, 2015, vol115, pp285-291.

[22] Marino F, De Ruvo P, De Ruvo G, Nitti M, Stella E. HiPER 3-D: An Omnidirectional Sensor for High Precision Environmental 3D Reconstruction. IEEE Transactions on Industrial Electronics 2012, 59(1): 579-91.

[23] Ukida H, Yamato N, Tanimoto Y, Sano T, Yamamoto H. Omnidirectional $3 \mathrm{~d}$ measurement by hyperbolic mirror cameras and pattern projection. In: IEEE International Instrumentation and Measurement Technology Conference, Victoria, USA, May 1215, 2008. pp. 365-70.

[24] Zhang C, Xu J, Xi N, Jia YY, and Li WX. Development of an omni-directional 3D camera for robot navigation. In: IEEE/ASME International Conference on AdvancedIntelligent Mechatronics, Kaohsiung, China, July 11-14, 2012. p. 262-67.

[25] Crdova-Esparza D M, Gonzalez-Barbosa J J, Hurtado-Ramos J B, and Ornelas-Rodriguez F J. A panoramic 3D reconstruction system based on the projection of patterns, International Journal of Advanced Robotic Systems, 2014, 11:55. doi: 10.5772/58227

[26] Xiong L D, and Jia S H. Phase-error analysis and elimination for nonsinusoidal waveforms in Hilbert transform digital-fringe projection profilometry. Optics Letters. 2009, 34(15), 23632365.

[27] Huang P S and Zhang S. Fast three-step phase-shifting algorithm. Applied Optics, 2006, 45(21), 5086-5091.

[28] Chen R, Xu J, Chen K, Chen H P, Chen K. A high-accuracy 3D projection system for fastener assembly. The 5th Annual IEEE International Conference on Cyber Technology in Automation, Control and Intelligent Systems Shenyang, China, June 8-12, 2015, pp.965-971

[29] Liu K, Wang Y C, Lau D L.,Hao Q, and Hassebrook L G. Dual-frequency pattern scheme for high-speed 3-D shape measurement, Optics Express, 2010, 18(5), 5229-5244

[30] Bouguet J Y. Camera calibration toolbox for Matlab. [Online]. Available: $h t t p: / / w w w . v i s i o n . c a l t e c h . e d u /$ bouguet $/$ calib $_{d}$ oci 